\title{
Perplexity of \\ n-gram and dependency language models
}

Martin Popel, David Mareček ÚFAL, Charles University in Prague

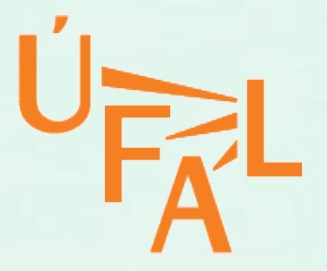

TSD, 13th International Conference on Text, Speech and Dialogue September 8, 2010, Brno 


\section{Outline}

- Language Models (LM)

- basics

- design decisions

- Post-ngram LM

- Dependency LM

- Evaluation

- Conclusion \& future plans 


\section{Language Models - basics}

$P(s)=?$

$\mathrm{P}($ The dog barked again $)$ 


\section{Language Models - basics}

$\mathrm{P}(\mathrm{s})=?$

$\mathrm{P}($ The dog barked again $)>\mathrm{P}($ The dock barked again $)$ 


\section{Language Models - basics}

$P(s)=P\left(w_{1}, w_{2}, \ldots w_{m}\right)$

$\mathrm{P}($ The dog barked again $)=$

$P\left(w_{1}=\right.$ The,$w_{2}=$ dog,$w_{3}=$ barked,$w_{4}=$ again $)$ 


\section{Language Models - basics}

$P(s)=P\left(w_{1}, w_{2}, \ldots w_{m}\right)=P\left(w_{1}\right) P\left(w_{2} \mid w_{1}\right) \ldots P\left(w_{m} \mid w_{1}, \ldots, w_{m-1}\right)$

\section{Chain rule}

$\mathrm{P}($ The dog barked again $)=$

$\mathrm{P}\left(\mathrm{w}_{1}=\right.$ The $)$.

$P\left(w_{2}=\operatorname{dog} \quad \mid w_{1}=\right.$ The $)$.

$\mathrm{P}\left(\mathrm{w}_{3}=\right.$ barked $\mid \mathrm{w}_{1}=$ The, $\mathrm{w}_{2}=$ dog $)$.

$\mathrm{P}\left(\mathrm{w}_{4}=\right.$ again $\mid \mathrm{w}_{1}=$ The, $\mathrm{w}_{2}=$ dog, $\mathrm{w}_{3}=$ barked $)$ 


\section{Language Models - basics}

$P(s)=P\left(w_{1}, w_{2}, \ldots w_{m}\right)=P\left(w_{1}\right) P\left(w_{2} \mid w_{1}\right) \ldots P\left(w_{m} \mid w_{1}, \ldots, w_{m-1}\right)$

\section{Changed notation}

$P($ The dog barked again $)=$

$P\left(w_{i}=\right.$ The $\left.\mid i=1\right)$.

$P\left(w_{i}=\operatorname{dog} \quad \mid i=2, w_{i-1}=\right.$ The $)$.

$P\left(w_{i}=\right.$ barked $\mid i=3, w_{i-2}=$ The,$w_{i-1}=$ dog $)$.

$\mathrm{P}\left(\mathrm{w}_{\mathrm{i}}=\right.$ again $\mid \mathrm{i}=4, \mathrm{w}_{\mathrm{i}-3}=$ The, $\mathrm{w}_{\mathrm{i}-2}=$ dog, $\mathrm{w}_{\mathrm{i}-1}=$ barked $)$ 


\section{Language Models - basics}

$$
P(s)=P\left(w_{1}, w_{2}, \ldots w_{m}\right)=P\left(w_{1}\right) P\left(w_{2} \mid w_{1}\right) \ldots P\left(w_{m} \mid w_{1}, \ldots, w_{m-1}\right)
$$

\section{Artificial start-of-sentence token}

$\mathrm{P}($ The dog barked again $)=$

$\mathrm{P}\left(\mathrm{w}_{\mathrm{i}}=\right.$ The $\quad \mathrm{i}=1, \mathrm{w}_{\mathrm{i}-1}=$ NONE $)$.

$P\left(w_{i}=\operatorname{dog} \quad \mid i=2, w_{i-2}=\right.$ NONE,$w_{i-1}=$ The $) \cdot$

$\mathrm{P}\left(\mathrm{w}_{\mathrm{i}}=\right.$ barked $\mid \mathrm{i}=3, \mathrm{w}_{\mathrm{i}-3}=$ NONE, $\mathrm{w}_{\mathrm{i}-2}=$ The, $\mathrm{w}_{\mathrm{i}-1}=$ dog $) \cdot$

$\mathrm{P}\left(\mathrm{w}_{\mathrm{i}}=\right.$ again $\mid \mathrm{i}=4, \mathrm{w}_{\mathrm{i}-4}=$ NONE, $\mathrm{w}_{\mathrm{i}-3}=$ The, $\mathrm{w}_{\mathrm{i}-2}=$ dog, $\mathrm{w}_{\mathrm{i}-1}=$ barked $)$ 


\section{Language Models - basics}

$$
P(s)=P\left(w_{1}, w_{2}, \ldots w_{m}\right)=P\left(w_{1}\right) P\left(w_{2} \mid w_{1}\right) \ldots P\left(w_{m} \mid w_{1}, \ldots, w_{m-1}\right)
$$

\section{Position backoff}

$\mathrm{P}($ The dog barked again $) \approx$

$\mathrm{P}\left(\mathrm{w}_{\mathrm{i}}=\right.$ The $\mid \quad \mathrm{w}_{\mathrm{i}-1}=$ NONE $)$.

$P\left(w_{i}=\operatorname{dog} \quad \mid \quad w_{i-2}=\right.$ NONE,$w_{i-1}=$ The $)$.

$\mathrm{P}\left(\mathrm{w}_{\mathrm{i}}=\right.$ barked $\mid \quad \mathrm{w}_{\mathrm{i}-3}=$ NONE, $\mathrm{w}_{\mathrm{i}-2}=$ The, $\mathrm{w}_{\mathrm{i}-1}=$ dog $)$.

$\mathrm{P}\left(\mathrm{w}_{\mathrm{i}}=\right.$ again $\mid \mathrm{w}_{\mathrm{i}-4}=$ NONE, $\mathrm{w}_{\mathrm{i}-3}=$ The, $\mathrm{w}_{\mathrm{i}-2}=$ dog, $\mathrm{w}_{\mathrm{i}-1}=$ barked $)$ 


\section{Language Models - basics}

$$
P(s)=P\left(w_{1}, w_{2}, \ldots w_{m}\right) \approx \prod_{i=1 . . m} P\left(w_{i} \mid w_{i-1}\right)
$$

\section{History backoff (bigram LM)}

$\mathrm{P}($ The dog barked again $) \approx$

$\mathrm{P}\left(\mathrm{w}_{\mathrm{i}}=\right.$ The $\mid \quad \mathrm{w}_{\mathrm{i}-1}=$ NONE $)$.

$P\left(w_{i}=\operatorname{dog} \quad\right.$ ।

$\mathrm{W}_{\mathrm{i}-1}=$ The $) \cdot$

$P\left(w_{i}=\right.$ barked I

$\left.\mathrm{W}_{\mathrm{i}-1}=\operatorname{dog}\right)$.

$\mathrm{P}\left(\mathrm{w}_{\mathrm{i}}=\right.$ again $\quad$ |

$\mathrm{w}_{\mathrm{i}-1}=$ barked ) 


\section{Language Models - basics}

$P(s)=P\left(w_{1}, w_{2}, \ldots w_{m}\right) \approx \prod_{i=1 . . m} P\left(w_{i} \mid w_{i-2}, w_{i-1}\right)$

\section{History backoff (trigram LM)}

$\mathrm{P}($ The dog barked again $) \approx$

$\mathrm{P}\left(\mathrm{w}_{\mathrm{i}}=\right.$ The $\quad \mathrm{w} \mathrm{w}_{\mathrm{i}-2}=$ NONE, $\mathrm{w}_{\mathrm{i}-1}=$ NONE $)$.

$P\left(w_{i}=\operatorname{dog} \quad \mid \quad w_{i-2}=\right.$ NONE,$w_{i-1}=$ The $)$.

$P\left(w_{i}=\right.$ barked $\mid$

$\mathrm{w}_{\mathrm{i}-2}=$ The, $\left.\mathrm{w}_{\mathrm{i}-1}=\operatorname{dog}\right)$.

$\mathrm{P}\left(\mathrm{w}_{\mathrm{i}}=\right.$ again $\quad$ |

$\mathrm{w}_{\mathrm{i}-2}=$ dog, $\mathrm{w}_{\mathrm{i}-1}=$ barked ) 


\section{Language Models - basics}

$P(s)=P\left(w_{1}, w_{2}, \ldots w_{m}\right) \approx \prod_{i=1 . . m} P\left(w_{i} \mid w_{i-2}, w_{i-1}\right)$

In general:

$\Pi_{i=1 . . m} P\left(w_{i} \mid h_{i}\right)$

$\mathbf{h}_{\mathrm{i}}$... context (history) of word $\mathrm{w}_{\mathrm{i}}$ 


\section{Language Models - design decisions}

1) How to factorize $P\left(w_{1}, w_{2}, \ldots w_{m}\right)$ into $\prod_{i=1 . . m} P\left(w_{i} \mid h_{i}\right)$, i.e. what word-positions will be used as the context $\mathbf{h}_{\mathbf{i}}$ ?

2) What additional context information will be used (apart from word forms), e.g. stems, lemmata, POS tags, word classes,...?

3) How to estimate $P\left(w_{i} \mid h_{i}\right)$ from the training data? Which smoothing technique will be used? (Good-Turing, Jelinek-Mercer, Katz, Kneser-Ney,...) Generalized Parallel Backoff etc. 


\section{Language Models - design decisions}

1) How t i.e. wha

this work

$\left.n / w_{1}, w_{2}, \ldots w_{m}\right)$ into $\Pi_{i=1 . . m} P\left(w_{i} \mid h_{i}\right)$, itions will be used as the context $\mathbf{h}_{\mathbf{i}}$ ?

2) What add context information will be used (apart from d forms), e.g. stems, mmata, POS tags, word classes,...?

3) How to estimate $P\left(w_{i} \mid h_{i}\right)$ from the training data?

Linear interpolation que will be used? Weights trained by EM ercer, Katz, Kneser-Ney,...) Generalized Parallel Backoff etc. 


\section{Language Models - design decisions}

1) How t i.e. wha

this work tions will $\mathbf{h}_{\mathrm{i}}=\mathrm{w}_{\mathrm{i}-\mathrm{n}+1}, \ldots, \mathrm{w}_{\mathrm{i}-1}$

(n-gram-based LMs)

2) What add context information will used (apart from d forms), e.g. stems, mmata, POS tags, word es,...?

3) How to estimate $P\left(w_{i} \mid h_{i}\right)$ from the th other ata?

Linear interpolation que will be papers Weights trained by EM ercer, Kat $y, \ldots)$ Generalized Parallel Backoff etc. 


\section{Outline}

- Language Models (LM)

- basics

- design decisions

- Post-ngram LM

- Dependency LM

- Evaluation

- Conclusion \& future plans 


\section{Post-ngram LM}

In general:

$P(s)=P\left(w_{1}, w_{2}, \ldots w_{m}\right) \approx \prod_{i=1 . . m} P\left(w_{i} \mid h_{i}\right)$

$h_{i} \ldots$ context (history) of word $w_{i}$

left-to-right factorization order

Bigram LM:

$\mathbf{h}_{\mathrm{i}}=\mathrm{w}_{\mathrm{i}-1}$

(one previous word)

Trigram LM:

$h_{i}=w_{i-2}, w_{i-1}$

(two previous words) 


\section{Post-ngram LM}

In general:

$\mathrm{P}(\mathrm{s})=\mathrm{P}\left(\mathrm{w}_{1}, \mathrm{w}_{2}, \ldots \mathrm{w}_{\mathrm{m}}\right) \approx \prod_{\mathrm{i}=1 . . \mathrm{m}} \mathrm{P}\left(\mathrm{w}_{\mathrm{i}} \mid \mathbf{h}_{\mathrm{i}}\right)$

$h_{i} \ldots$ context (history) of word $w_{i}$

left-to-right factorization order

Bigram LM:

$\mathbf{h}_{\mathrm{i}}=\mathrm{w}_{\mathrm{i}-1}$

(one previous word)

Trigram LM:

$\mathbf{h}_{\mathrm{i}}=\mathrm{w}_{\mathrm{i}-2}, \mathrm{w}_{\mathrm{i}-1}$

(two previous words)

right-to-left factorization order

Post-bigram LM: $\quad \mathbf{h}_{\mathrm{i}}=\mathrm{w}_{\mathrm{i}+1}$

(one following word)

Post-trigram LM: $\mathbf{h}_{\mathrm{i}}=\mathrm{w}_{\mathrm{i}+1}, \mathrm{w}_{\mathrm{i}+2}$

(two following words) 


\section{Post-ngram LM}

In general:

$\mathrm{P}(\mathrm{s})=\mathrm{P}\left(\mathrm{w}_{1}, \mathrm{w}_{2}, \ldots \mathrm{w}_{\mathrm{m}}\right) \approx \prod_{\mathrm{i}=1 . . \mathrm{m}} \mathrm{P}\left(\mathrm{w}_{\mathrm{i}} \mid \mathbf{h}_{\mathrm{i}}\right)$

$\mathbf{h}_{\mathrm{i}} \ldots$ context (history) of word $w_{i}$

left-to-right factorization order

Bigram LM:

$\mathbf{h}_{\mathrm{i}}=\mathrm{w}_{\mathrm{i}-1}$

(one previous word)

Trigram LM:

$\mathbf{h}_{\mathrm{i}}=\mathrm{w}_{\mathrm{i}-2}, \mathrm{w}_{\mathrm{i}-1}$

(two previous words)

right-to-left factorization order

Post-bigram LM: $\mathbf{h}_{\mathrm{i}}=\mathrm{w}_{\mathrm{i}+1} \quad$ (one following word)

$P($ The dog barked again $)=P($ again $\mid$ NONE $) \cdot P($ barked $\mid$ again $) \cdot$

$P($ dog $\mid$ barked $) \cdot P($ The $\mid$ dog $)$ 


\section{Outline}

- Language Models (LM)

- basics

- design decisions

- Post-ngram LM

- Dependency LM

- Evaluation

- Conclusion \& future plans 


\section{Dependency LM}

- exploit the topology of dependency trees

The dog barked again 


\section{Dependency LM}

- exploit the topology of dependency trees

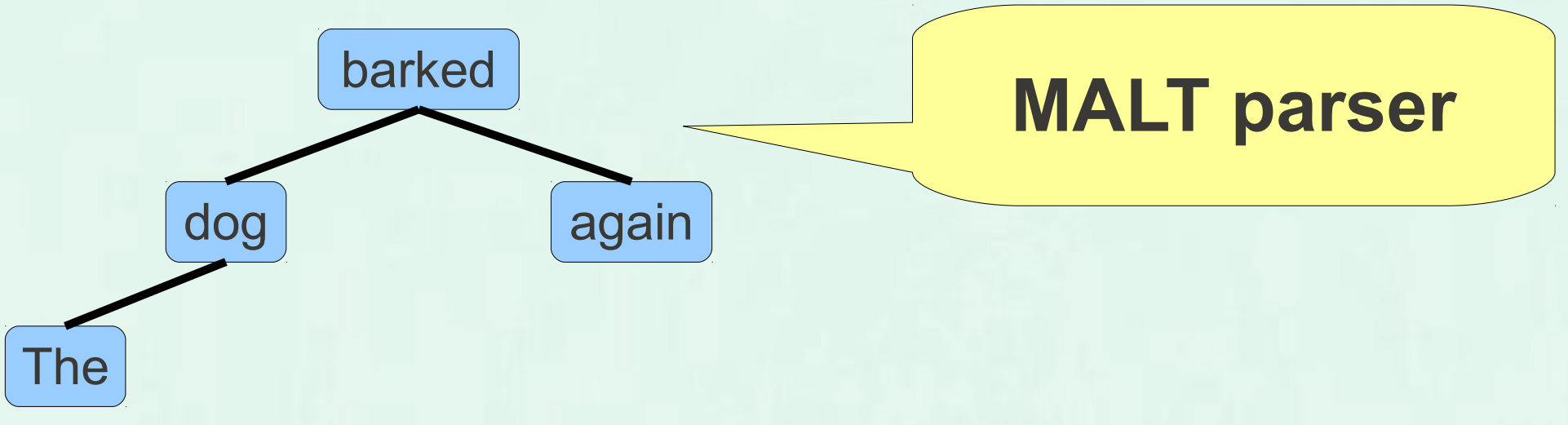




\section{Dependency LM}

- exploit the topology of dependency trees

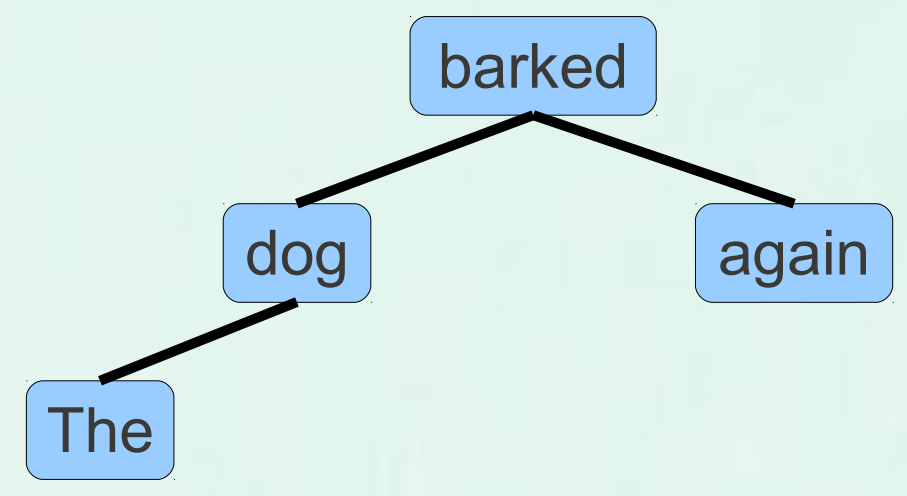

$$
\begin{aligned}
P(\text { The dog barked again })= & P(\text { The } \mid \text { dog }) \cdot P(\text { dog } \mid \text { barked }) \cdot \\
& P(\text { barked } \mid \text { NONE }) \cdot P(\text { again } \mid \text { barked })
\end{aligned}
$$

$\mathbf{h}_{\mathrm{i}}=\operatorname{parent}\left(\mathrm{w}_{\mathrm{i}}\right)$ 


\section{Dependency LM Long distance dependencies}

The dog $I$ heard last night barked again

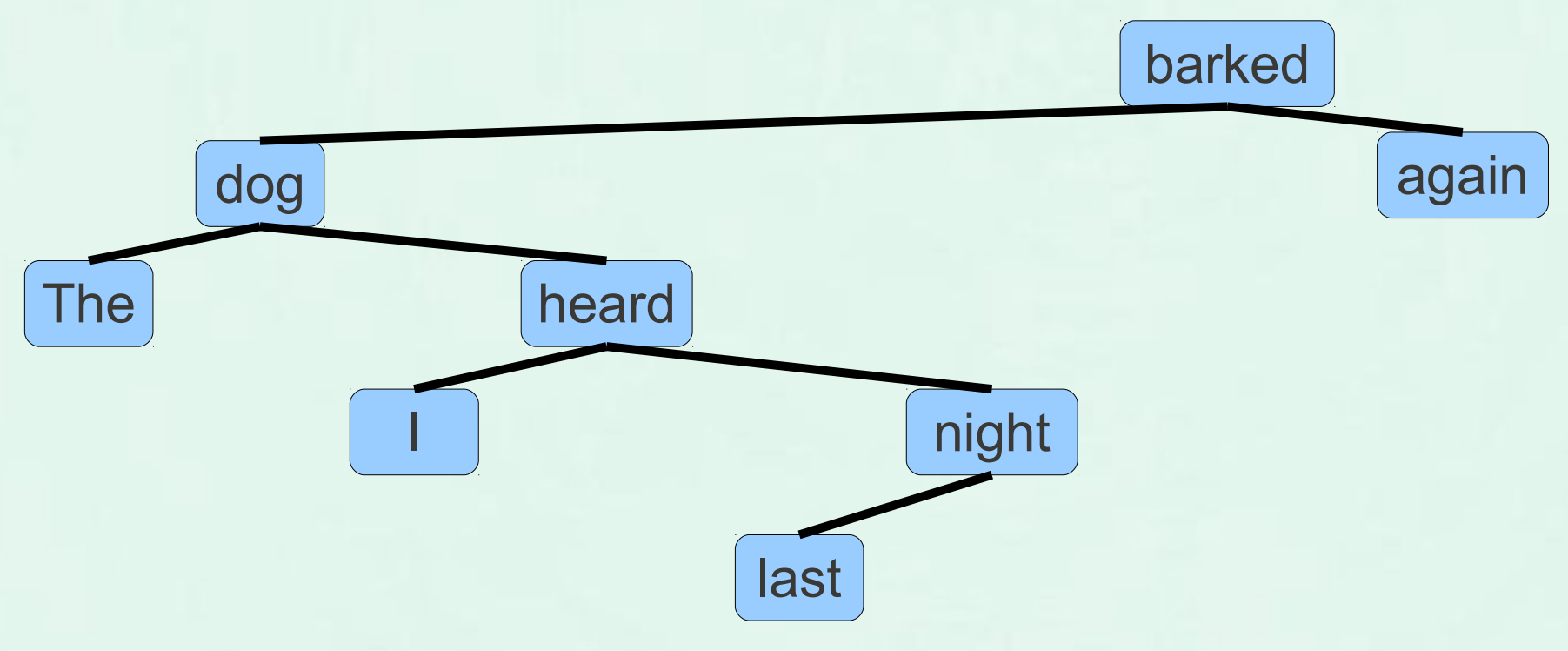




\section{Dependency LM Motivation for usage}

- How can we know the dependency structure without knowing the word-forms? 


\section{Dependency LM Motivation for usage}

- How can we know the dependency structure without knowing the word-forms?

- For example in tree-to-tree machine translation.
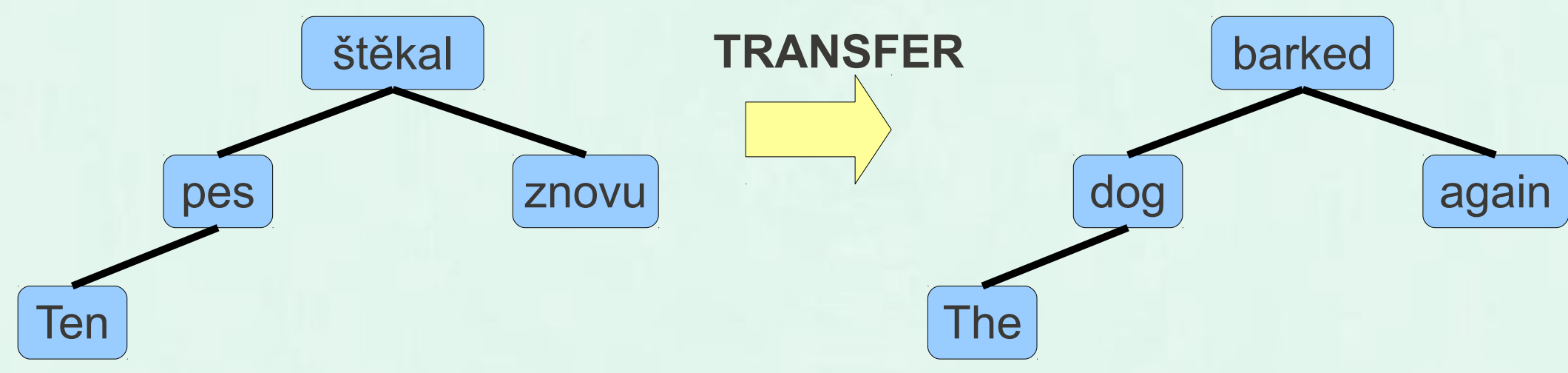

ANALYSIS

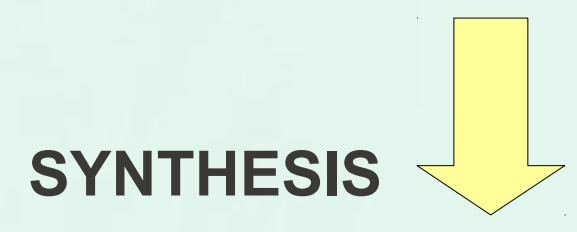

Ten pes štěkal znovu

The dog barked again 


\section{Dependency LM Examples}

- Model wp word form of parent

$P($ The dog barked again $)=$

\begin{tabular}{l|l|}
$P($ The $\mid$ dog & ) \\
$P($ dog $\mid$ barked & ) \\
$P($ barked $\mid$ NONE & ). \\
$P\left(\begin{array}{l|ll}\text { again } & \text { barked }\end{array}\right.$
\end{tabular}




\section{Dependency LM Examples}

- Model wp, wg word form of parent, word form of grandparent

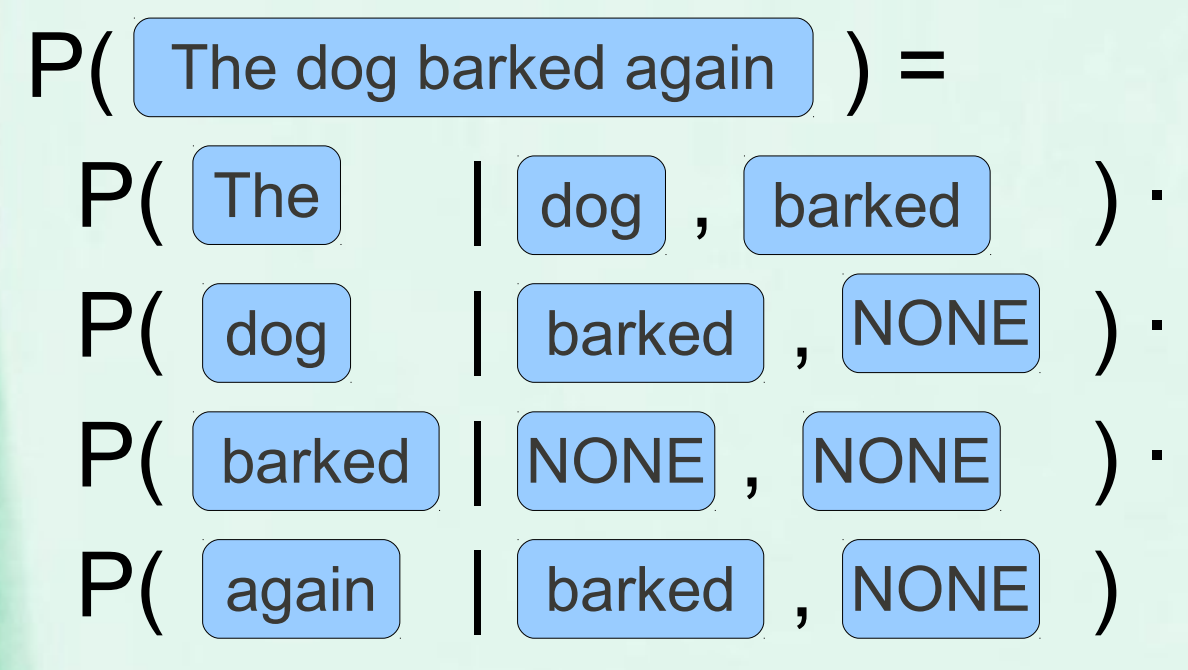




\section{Dependency LM Examples}

- Model E, wp edge direction, word form of parent
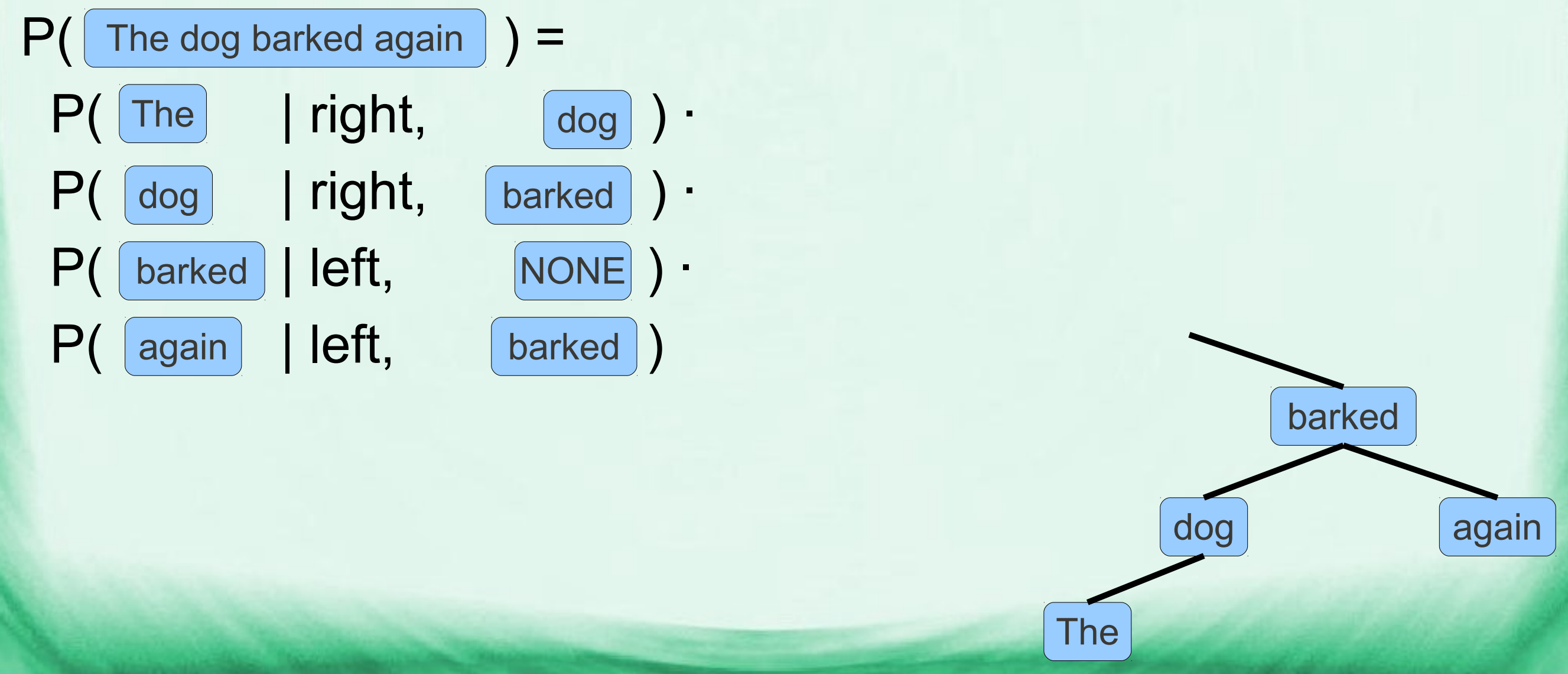


\section{Dependency LM Examples}

- Model C, wp number of children, word form of parent

$P($ The dog barked again $)=$

\begin{tabular}{|c|c|c|}
\hline$P$ ( The & 0 & dog \\
\hline$P(\operatorname{dog}$ & | 1 & barked \\
\hline $\mathrm{P}($ barked & 2 & NONE \\
\hline $\mathrm{P}($ again & 0 & barked \\
\hline
\end{tabular}

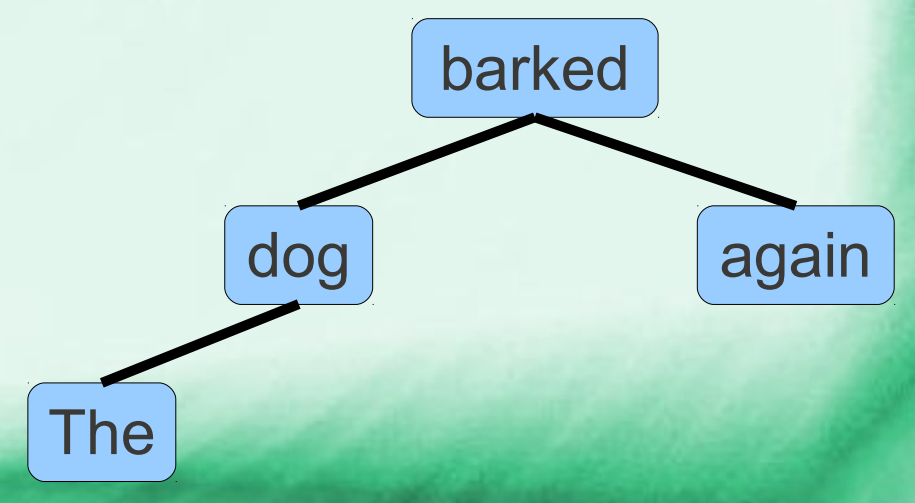




\section{Dependency LM Examples}

- Model N, wp the word is $\mathrm{N}^{\text {th }}$ child of its parent, word form of parent

$P($ The dog barked again $)=$

$P($ The $\mid 1$,
$P($ dog $\mid 1, \quad$ barked $)$.
$P($ barked $\mid 1$,
$P($ again $\mid 2$,




\section{Dependency LM Examples of additional context information}

- Model tp, wp

POS tag of parent, word form of parent

$P($ The dog barked again $)=$

$P($ The INN, dog $)$.

$P($ dog IVBD, barked ) .

$P($ barked | NONE, NONE $)$.

$\mathrm{P}($ again IVBD, barked $)$ 


\section{Dependency LM Examples of additional context information}

- Model tp, wp

POS tag of parent, word form of parent

$P($ The dog barked again $)=$ $P($ The INN, dog $)$. $P($ dog IVBD, barked $)$. $\mathrm{P}($ barked | NONE, NONE $)$. $P($ again |VBD, barked ) naïve tagger assignes the most frequent tag for a given word 


\section{Dependency LM Examples of additional context information}

- Model Tp, wp coarse-grained POS tag of parent, word form of parent

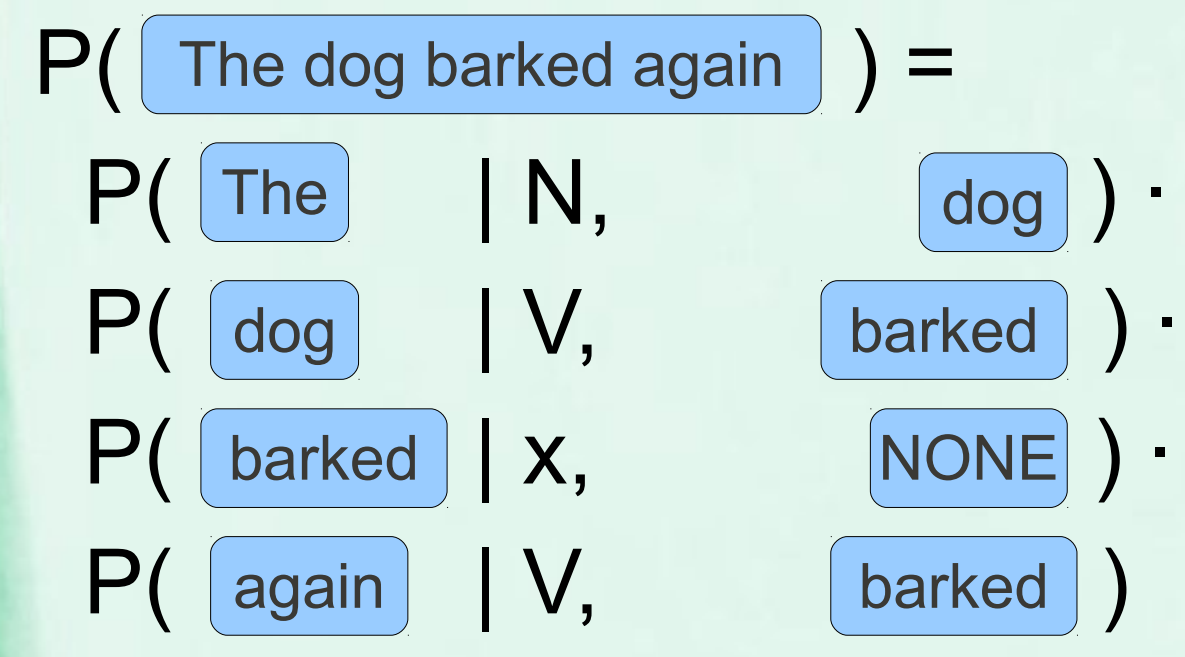

\begin{tabular}{|c|c|c|}
\hline$P$ ( The & $\mathrm{N}$ & $\operatorname{dog})$ \\
\hline$P($ dog & IV & barked ) \\
\hline $\mathrm{P}($ barked & $\mathrm{x}$ & NONE \\
\hline $\mathrm{P}($ again & $\mathrm{V}$ & barked ) \\
\hline
\end{tabular}




\section{Dependency LM Examples of additional context information}

- Model E, C, wp, N

edge direction, \# children, word form of parent, word is $\mathrm{N}^{\text {th }}$ child of its parent

$P($ The dog barked again $)=$

$\mathrm{P}$ ( The | right, $0, \operatorname{dog}, 1)$.

$\mathrm{P}($ dog | right, 1, barked, 1).

$P($ barked | left, 2, NONE, 1) ·

$P($ again | left, 0 , barked, 2) 


\section{Outline}

- Language Models (LM)

- basics

- design decisions

- Post-ngram LM

- Dependency LM

- Evaluation

- Conclusion \& future plans 


\section{Evaluation}

- Train and test data from CoNLL 2007 shared task

- 7 languages: Arabic, Catalan, Czech, English (450 000 tokens, 3 \% OOV), Hungarian, Italian (75 000 tokens), and Turkish (26 \% OOV)

- Cross-entropy $=-(1 /|\mathrm{T}|) \Sigma_{\mathrm{i}=1 . .|\mathrm{T}|} \log _{2} \mathrm{P}\left(\mathrm{w}_{\mathrm{i}} \mid \mathbf{h}_{\mathrm{i}}\right)$, measured on the test data $T$

- Perplexity $=2$ Cross-entropy

- Lower perplexity better LM

- Baseline ... trigram LM

- 4 experimental settings: PLAIN, TAGS, DEP, DEP+TAGS 


\section{Evaluation}

\section{normalized} perplexity

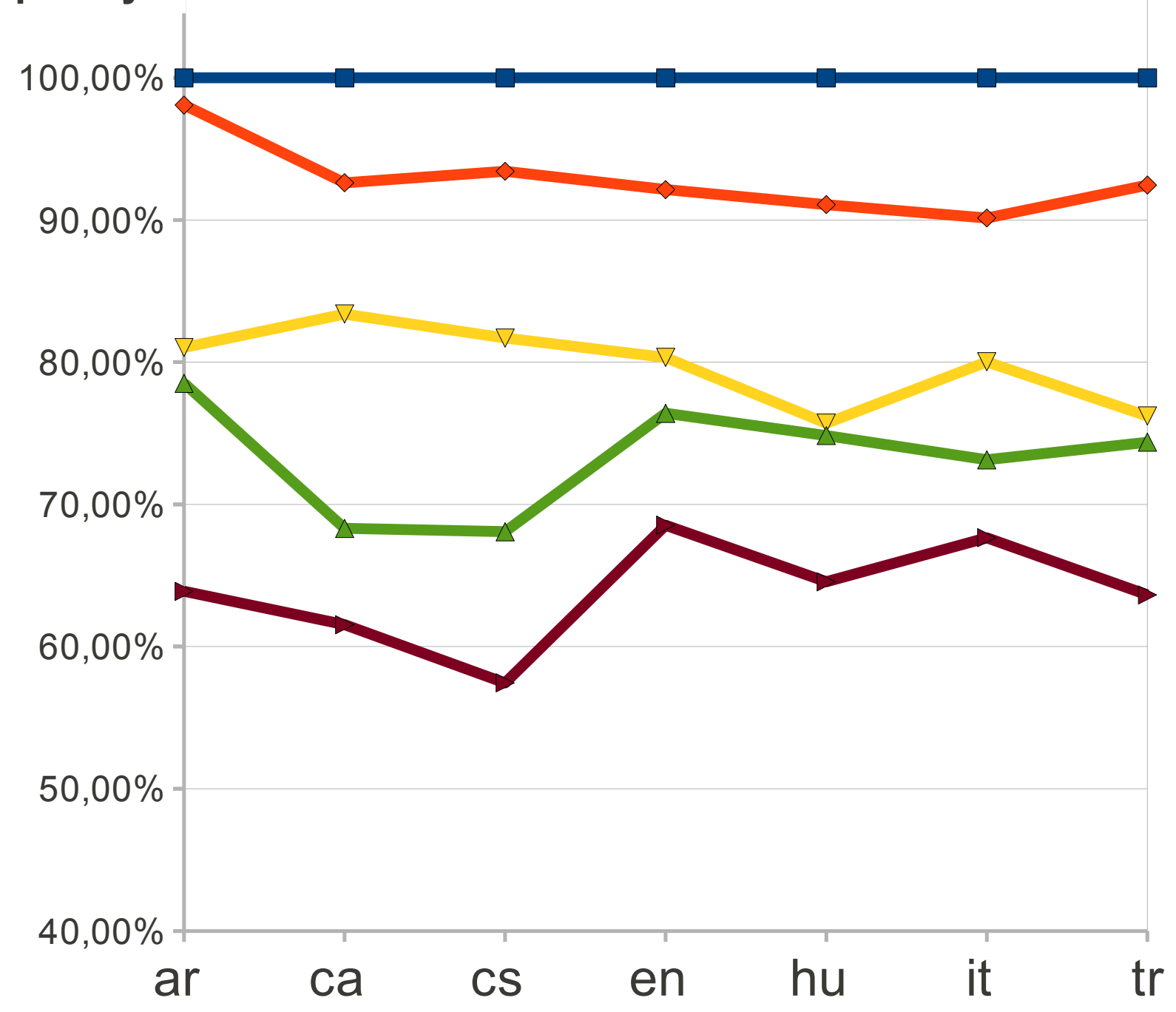

$-\mathrm{w}-1, \mathrm{w}-2$ (BASELINE)

$\Leftrightarrow \mathrm{w}+1, \mathrm{w}+2$ (PLAIN)

$\nabla \mathrm{T}+1, \mathrm{t}+1, \mathrm{I}+1, \mathrm{w}+1, \mathrm{~T}+2, \mathrm{t}+2, \mathrm{I}+2, \mathrm{w}+2$ (TAGS)

$\Rightarrow E, C, w p, N, w g$ (DEP)

$\Rightarrow$ E,C,Tp,tp,N,Ip, wp, Tg,tg,lg (DEP+TAGS) 


\section{Conclusion}

Findings confirmed for all seven languages

- Post-trigram better than trigram Post-bigram better than bigram

- Additional context (POS \& lemma) helps

- Dependency structure helps even more

- The best perplexity achieved with
Improvement over baseline for English
PLAIN $\quad 8 \%$

TAGS $20 \%$

DEP $24 \%$

DEP+TAGS $31 \%$ 


\section{Future plans}

- Investigate the reason for better post-ngram LM perplexity

- Extrinsic evaluation

- Post-ngram LM in speech recognition

- Dependency LM in tree-to-tree machine translation

- Better smoothing using Generalized Parallel Backoff

- Bigger LM for real applications 
Thank you 